REVISTA

MEXICANA DE

ECONOMÍA Y

FINANZAS

REMEF

(THE MEXICAN JOURNAL OF

ECONOMICS AND FINANCE
Revista Mexicana de Economía y Finanzas, Nueva Época

Volumen 16 Número 1, Enero - Marzo 2021, pp. 1-15, e563

DOI: https://doi.org/10.21919/remef.v16i1.563

(Recibido: 25/mayo/2020, aceptado: 27/agosto/2020, publicado: 26/octubre/2020)

\title{
Dinámica anticipada del PIB trimestral en México ante shocks negativos derivados de factores debidos a la crisis sanitaria del covid-19
}

\author{
Gustavo Cabrera González ${ }^{1}$ - Universidad de Guadalajara, México \\ Adrián de León Arias ${ }^{2}$ - Universidad de Guadalajara, México
}

Este artículo presenta los efectos anticipados en la tasa de crecimiento del producto interno bruto (PIB) trimestral de México debido a perturbaciones (shocks) que se espera la afecten en los próximos trimestres, en el contexto de los impactos del covid-19. Con este objetivo, se identifica la especificación econométrica markoviana autorregresiva de mejor ajuste a los datos de la dinámica del crecimiento del PIB de Q1-1960 a Q42019, bajo la condición de cuatro regímenes diferentes, dados por cambios en la media y por la volatilidad de la tasa de crecimiento. Posteriormente se introducen shocks negativos que asimilan diversos efectos macroeconómicos derivados del impacto económico del covid-19. En este ejercicio de simulación, la tasa de crecimiento del PIB puede permanecer en recesión de dos a seis trimestres, lo cual depende de la magnitud y persistencia que podrían tener los shocks negativos y a los posibles efectos estabilizadores que incidirán en la producción en México. Esta contribución ofrece un modelo para cuantificar efectos macroeconómicos ante eventos significativos e inesperados, con la limitación de que tales eventos podrían alejarse de distribuciones de probabilidad convencionales.

JEL Classification: O4, C24, C53.

Palabras clave: PIB, parámetros markovianos, pronóstico, economía mexicana.

\section{Anticipated Dynamics of Quarterly GDP in Mexico in the Face of Negative Shocks Arising from Factors due to the Covid-19 Health Crisis}

This article presents the anticipated effects in terms of Mexico's Gross National Product (GNP) quarterly growth rates due to shocks which are expected to impact those quarterly growth rates in the context of the covid-19 macroeconomics impacts. As econometric procedures, we identify a Markovian autoregressive of best-fit to data on the dynamics of those quarterly growth rate from Q1-1960 to Q4-2019, under four statistical states, given by different means and volatilities in growth rates. After that, we introduce some negative shocks that are equivalent to several macroeconomic effects related to covid-19. Through this econometric simulation exercise, we found that the GNP growth rate could be negative during a period from two to six yearly quarter, depending on the magnitude and persistence of negative shocks and the eventual stabilizing effects that could occur in the Mexican economy. This contribution offers an econometric model to estimate the impacts of relevant and unexpected macroeconomics events even when such impacts could not be described under conventional probability distributions.

Clasificación JEL: O4, C24, C53.

Keywords: GNP, Markov switching, forecasting, Mexican economy.

${ }^{1}$ Autor para códigos y correspondencia sobre este artículo al correo gustavo.cgonzalez@academicos.udg.mx

2 Los autores agradecen a la Dra. María del Rosario Cervantes Martínez por sus recomendaciones y comentarios a este trabajo, a la Mtra. Laura Selene Vielmas García, y Lic. J. David Rodríguez Álvarez por el apoyo en la edición.

*Sin fuente de financiamiento para el desarrollo de la investigación 


\section{Introducción}

La modelación de la dinámica de los ciclos económicos que presentamos en este trabajo está fundamentada en el principio seminal de análisis econométrico de Burns y Mitchell (1946) sobre asimetrías en la dinámica temporal de las variables económicas y sus respuestas en las diferentes fases de los ciclos económicos. Si interpretamos esta definición en sólo dos fases o estados del ciclo económico, podemos esperar caídas abruptas en la tasa de crecimiento y alta volatilidad en recesión durante pocos periodos de tiempo, así como periodos más largos de expansión con tasas de crecimiento positivas y baja volatilidad. Por lo tanto, se espera que en cada fase el impacto de las perturbaciones o shocks del ciclo sea de diferente magnitud y duración, y que haya distintas probabilidades de transición entre fases.

Por otro lado, en la literatura se empiezan a publicar documentos de trabajo sobre los efectos del covid-19 en los mercados financieros, como los de Günay (2020a, 2020b) y Zeren y Hizarci (2020), y se documentan efectos económicos a escala global, como la generación de incertidumbre, en Sulkowski (2020), Ozili y Thankom (2020), Baker et al. (2020) y Fernandes (2020). En este artículo analizamos la dinámica del PIB trimestral de México ante shocks negativos que asimilan los efectos macroeconómicos significativos asociados a la crisis sanitaria del covid-19; los examinamos a través de cambios y transiciones en el término de error de la ecuación econométrica.

En esta investigación, sin importar cuál sea la especificación econométrica del modelo ni cuáles sean las variables exógenas que se pudieran elegir, la estabilidad de la variable dependiente (crecimiento trimestral del PIB) es afectada aleatoriamente por disturbios (shocks) que tomarían en cuenta todas las variables predeterminadas excluidas (como la recesión económica de Estados Unidos, el desempleo en México, el cierre de actividades económicas, la inestabilidad financiera y política, etc.), los errores de medición y eventos fortuitos que no puede controlar el investigador. En este sentido, se asume que los shocks negativos considerados en este trabajo se asocian principalmente a los efectos de la crisis sanitaria del covid-19. Es importante mencionar que algunas posibles magnitudes y la persistencia de shocks negativos en el contexto actual no necesariamente provienen de una distribución normal. Sin embargo, son shocks realistas en el sentido en que lo plantean Hafner y Herwartz (2006).

El objetivo de este trabajo es medir el número de trimestres que le llevará a la tasa de crecimiento del PIB - después de shocks significativos en magnitud, negativos y posiblemente persistentes - alcanzar la estabilidad en cualquier fase del ciclo económico en que se pudiera encontrar en el futuro: recesión, estancamiento ${ }^{3}$ o expansión.

Este trabajo se organiza de la siguiente manera: en la primera sección presentamos estadística descriptiva de los datos del PIB de México de Q1-1960 a Q4-2019, que motivan los métodos econométricos de la segunda sección. En la tercera sección se reportan los resultados en que se sustentan las conclusiones del último apartado.

\footnotetext{
${ }^{3}$ Si bien no hay una definición consensada sobre el término estancamiento, aquí los definimos como aquel periodo cuyas tasas de crecimiento anuales están por debajo del tres por ciento por al menos cinco años.
} 


\section{Congruencia de modelación con parámetros markovianos con la dinámica de la tasa de crecimiento trimestral del PIB de México, 1960-2019}

En general, el modelado de las series de tiempo estacionarias en econometría se basa en dos estrategias de identificación. La primera se refiere a los modelos estructurales que utilizan determinantes (variables exógenas o explicativas); la segunda, a aquellos identificados por la propia dinámica autorregresiva de la serie de tiempo. Puesto que el objetivo de este artículo no es determinar cuál variable afecta el crecimiento del PIB de México sino inferir su crecimiento fuera de la muestra con base en la memoria de la propia serie aplicando shocks externos futuros que afectarán su estabilidad dinámica, recurrimos a modelos autorregresivos.

A partir del artículo seminal de Hamilton (1989), hay una amplia literatura que estudia series de tiempo económico-financieras con modelos de cambios de régimen basados en parámetros markovianos (véanse las compilaciones de Hamilton y Raj 2002, y Frühwirth-Schnatter 2006). Con base en lo anterior, planteamos la siguiente pregunta: ¿por qué modelar con parámetros markovianos las dinámicas de las series de tiempo económico-financieras de México?

En primer lugar, las series de tiempo largas, que cubren periodos de cambios en política monetaria, fiscal, crisis económicas o financieras, dejan cambios en los parámetros que caracterizan a la serie a través del tiempo, e incluso cambios estructurales que en muchas ocasiones no puede imponer en forma exógena el investigador ${ }^{4}$ (para series de producción y ciclos económicos en México véanse, por ejemplo, Mejía-Reyes et al. 2005 y 2010, y Cabrera y De León Arias 2019). Más aún, asumir que la media de un proceso o su volatilidad es la misma en todos los momentos del tiempo es un supuesto difícil de sostener empíricamente. Para ilustrar lo anterior, en la figura 1 presentamos los momentos de la media y la desviación estándar de submuestras de la tasa de crecimiento del PIB de México mostrando cambios en el tiempo.

En segundo lugar, la especificación de un modelo econométrico y sus inferencias asociadas determinan las conclusiones del estudio. Sin embargo, tales resultados podrían ser diferentes con una especificación alternativa. Por ejemplo, Boot y Pick (2017) pronostican el PIB de la economía de Estados Unidos con un modelo de tres regímenes y muestran que esta especificación tiene menos errores de pronóstico que los modelos de dos estados y de especificaciones con parámetros constantes en el tiempo. Por lo tanto, en este estudio - para controlar por algún supuesto erróneo en la especificación - se prueba una batería de múltiples especificaciones markovianas, que incluyen modelos con parámetros constantes, y se elige la mejor especificación de ajuste a los datos de la tasa de crecimiento del PIB de México de Q1-1960 a Q4-2019.

En tercer lugar, los procesos markovianos dejan mezclas de distribuciones de probabilidad que no necesariamente siguen una distribución normal; en algunas ocasiones pueden dejar colas anchas o largas, asimetrías y posible multimodalidad, como muestra la evidencia empírica de las series económico-financieras. En la figura 1 presentamos la distribución de probabilidad

\footnotetext{
${ }^{4}$ Una práctica común para modelar el crecimiento del PIB de México es imponer variables dicotómicas en las recesiones de 1995 y 2008-2009. Sin embargo, más adelante se mostrará que hay más cambios de régimen que los de estos años en particular.
} 
(determinada por el ajuste del kernel) de las tasas de crecimiento trimestrales del PIB de México de Q1-1960 a Q4-2019; en la gráfica se observa que los datos no siguen una distribución de probabilidad normal, sino que más bien se caracterizan por una mezcla de distribuciones con una larga cola a la izquierda y presentan multimodalidad. Más aún, la hipótesis nula de normalidad de la prueba JarqueBera (JB) es rechazada (véanse también las medidas de asimetría a la izquierda y la distribución en forma de pico por la curtosis).

\begin{tabular}{|l|r|}
\hline \multicolumn{2}{|c|}{ Muestral total } \\
\hline Número de trimestres & 239 \\
\hline Media & 0.9156 \\
\hline Desviación estándar & 1.2195 \\
\hline \multicolumn{1}{|c|}{ p-value (JB) } & 0.0010 \\
\hline Asimetría & - \\
\multicolumn{2}{|c|}{ Curtosis } \\
\hline \multicolumn{2}{|c|}{ Submuestras } \\
\hline Media 1960-1980 & 6.9879 \\
\hline $\begin{array}{l}\text { Desviación estándar } \\
1980 \text {-1994 }\end{array}$ & 1.5723 \\
\hline Media 2010-2019 & 0.3447 \\
\hline $\begin{array}{l}\text { Desviación estándar } \\
\text { 2010-2019 }\end{array}$ & 0.5459 \\
\hline
\end{tabular}

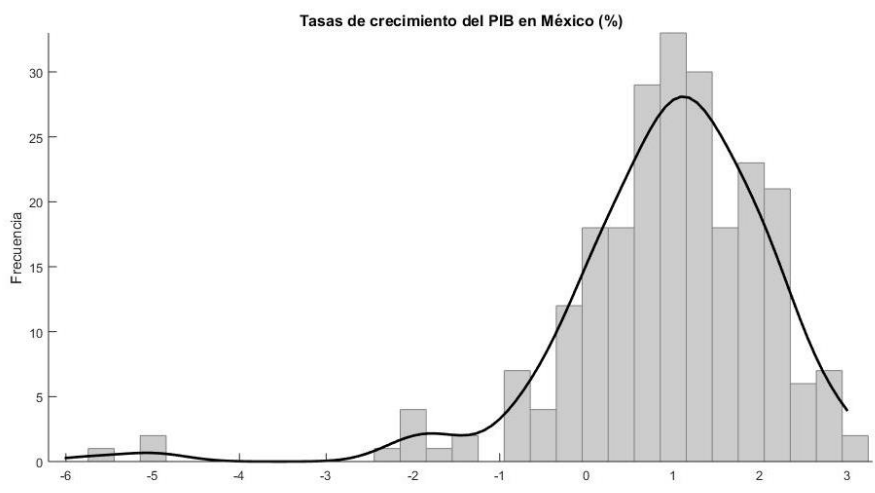

Figura 1. Estadística descriptiva de la tasa de crecimiento trimestral del PIB, 1960-2019 Fuente: https://data.oecd.org. El histograma y el ajuste del kernel de la distribución de probabilidad son elaboraciones propias.

\section{Modelación econométrica para elección del modelo}

En esta sección, con base en el análisis anterior, presentamos nuestra propuesta de especificación econométrica para estudiar el PIB trimestral de México de Q1-1960 a Q4-2019, así como el criterio de selección de la mejor especificación de ajuste a los datos.

\subsection{Propuesta de modelo econométrico autorregresivo con parámetros markovianos}

Sea $y_{t}$ la serie de tiempo estacionaria del crecimiento porcentual del PIB por tal, para todo $t$ se tiene: ${ }^{5}$

$$
\phi_{\left[S_{t}\right]}(L) y_{t}=\zeta_{\left[S_{t}\right]}+\sigma_{\left[S_{t}\right]} \varepsilon_{t} \text {, donde } \varepsilon_{t} \sim \operatorname{NIID}(0,1)
$$

\footnotetext{
${ }^{5}$ La notación y las definiciones están basadas en Frühwirth-Schnatter (2004) y fueron aplicadas antes en Cabrera et al. (2018) y Cabrera y De León (2019).
} 
Los parámetros autorregresivos $\phi_{\left[S_{t}\right]}$ dependen del estado o régimen, $L$ es el operador de rezagos para el polinomio autorregresivo de orden $(p)$, el intercepto $\zeta_{\left[S_{t}\right]}$ implicaría una media dependiente del estado y $\sigma_{\left[s_{t}\right]}$ captura la volatilidad del término de error por estado, lo cual haría que la volatilidad del proceso $y_{t}$ dependiera del régimen.

El indicador de los estados $S_{t}$ es una variable estocástica definida en un espacio discreto y finito $\{1, \ldots, K\}$ que captura la dependencia de los parámetros del régimen para todo $t$. Para la transición entre los estados se asume un proceso de Markov de primer orden, es decir, la probabilidad de transición del estado $k$ al estado $l$ está dada por $\xi_{\mathrm{kl}}=\operatorname{Pr}\left(\mathrm{S}_{\mathrm{t}}=\mathrm{l} \mid \mathrm{S}_{\mathrm{t}-1}=\mathrm{k}\right)$ para $t=$ $1, \ldots, T ;$ y los estados $k, l \in\{1, \ldots, K\}$.

Para modelar la tasa de crecimiento del PIB de México por la ecuación (1) se asume que el número de rezagos $(p)$ se define parsimoniosamente de cero a tres trimestres y el número de estados $(K)$ de uno a cuatro posibles regímenes, lo que implicaría un total de 16 posibles especificaciones autorregresivas.

En esta investigación, por la ventaja que nos da la metodología utilizada, los efectos de la crisis sanitaria del covid-19 no son modelados como variables independientes o exógenas, sino que se introducen a través del término de error $\left(\sigma_{\left[s_{t}\right]} \varepsilon_{t}\right)$ de la ecuación (1), el cual incluye dos términos: la volatilidad del shock dependiente del estado $\sigma_{\left[s_{t}\right]}$ y un término estocástico $\left(\varepsilon_{t}\right)$ proveniente de una distribución normal estándar distribuido idéntica e independientemente. A partir de dicho término de error se resumen los siguientes hechos para modelar los efectos de la crisis sanitaria:

1. En cada posible régimen o estado $(k)$ el término de error sigue una distribución de probabilidad normal con media de cero y varianza $\left(\sigma_{\left[S_{t}=k\right]}^{2}\right)$. Sin embargo, el total de la serie del término de error sigue una mezcla de distribuciones normales que no necesariamente deja otra distribución normal. En algunas ocasiones dicha mezcla de distribuciones podría dejar colas anchas, asimetría, curtosis y posible multimodalidad (véase Frühwirth-Schnatter, 2006).

2. En el término estocástico $\left(\varepsilon_{t}\right)$ se introducirán shocks negativos derivados de la crisis sanitaria del covid-19. Aunque ninguna de dichas perturbaciones proviene de una distribución normal estándar, serán escaladas por la varianza de cada régimen. Lo anterior porque se considerarán posibles cambios de régimen por la aplicación de shocks negativos (véanse las funciones de impulso-respuesta — no lineales por el cambio de régimen- en la sección 3.2).

3. En el término de error se considerarán dos posibles escenarios para introducir las perturbaciones:

i) Shocks negativos persistentes cada trimestre derivados de las consecuencias que se espera tenga la crisis sanitaria del covid-19, como recesión en Estados Unidos, altos niveles de desempleo en México, rebrotes del covid, cierres de actividades económicas e inestabilidad financiera y política, entre otros. Se espera que todos estos factores derivados de la crisis sanitaria afecten negativamente la tasa de crecimiento del PIB de México, por lo cual los shocks podrían llegar a ser de gran magnitud. En este trabajo consideramos shocks negativos de 2\%,4\%,6\% y 8\% decrecientes hasta cero cada trimestre (véase el primer escenario de la sección 3.2). 
ii) Un solo shock negativo de $2 \%, 4 \%, 6 \%$ y $8 \%$ en el primer trimestre, e inmediatamente después shocks provenientes de una distribución normal por régimen porque se esperan cambios de estado en el futuro y que las políticas estabilizadoras del Gobierno de México anulen los efectos negativos derivados de la crisis sanitaria del covid-19 (véase el segundo escenario de la sección 3.2).

En resumen, es importante mencionar que los efectos derivados de la crisis sanitaria del covid-19, en su conjunto, dejarán fuertes perturbaciones negativas fuera de una distribución normal, y quizá sean persistentes estos shocks. Por lo tanto, es limitado un supuesto de normalidad para los impulsos o shocks estándar de la literatura econométrica, dada la evidencia empírica de los acontecimientos de la crisis sanitaria. En tal sentido, en este trabajo aplicamos el concepto de shocks realistas sugerido por Hafner y Herwartz (2006).

\subsection{Elección de la mejor especificación de ajuste a los datos}

La identificación del modelo de mejor ajuste a los datos se lleva a cabo por medio de la simulación bayesiana sugerida por Frühwirth-Schnatter (2004) para determinar la especificación econométrica apropiada, sobre la cual se sustentarán los resultados de la siguiente parte. Con este objetivo se estima la probabilidad marginal de todos los modelos (16 en total) por métodos de simulación Monte Carlo (MCMC). Una vez encontrada la probabilidad marginal de cada especificación, se calcula el factor de Bayes contra la probabilidad marginal más alta. A partir de esta razón, en la Tabla 1 se reporta el logaritmo base 10 y se aplica la regla de selección de modelo sugerida por Kass y Raftery (1995) (véanse las dos últimas columnas). Por lo tanto, los modelos con evidencia en contra serían descartados ante el modelo que mejor se ajuste a los datos.

Al aplicar la regla mencionada, la especificación con mejor ajuste a los datos sugiere dos rezagos con cuatro estados $\left(\log _{10}=0\right)$. Es importante hacer notar que las especificaciones de un solo estado $(K=1)$ con parámetros constantes en el tiempo se rechazan con evidencia fuerte, de acuerdo con los resultados de la Tabla 1. Las únicas especificaciones que no se rechazarían con evidencia positiva (marcadas con subrayado) son tres estados y dos rezagos y la de cuatro estados y tres rezagos. Esto habrá de condicionar los resultados que se presentan en la siguiente sección.

Tabla 1. Factor de Bayes en $\log _{10}$ y regla de decisión

\begin{tabular}{|c|c|c|r|r|r|r|}
\hline $\mathbf{p} / \mathbf{K}$ & $\mathbf{1}$ & $\mathbf{2}$ & \multicolumn{1}{|c|}{$\mathbf{3}$} & \multicolumn{1}{|c|}{$\mathbf{4}$} & $\mathbf{B F}$ en $\mathbf{~} \mathbf{0 g}_{\mathbf{1 0}}$ & Evidencia en contra \\
\hline 0 & 54.4521 & 37.0506 & 23.3347 & 18.8241 & 1 a 3 & No vale más que una simple mención \\
\hline 1 & 44.2509 & 15.3613 & 6.6182 & 4.9529 & 3 a 20 & Positiva \\
\hline 2 & 44.6738 & 13.6189 & $\underline{2.8016}$ & $\mathbf{0 . 0 0 0 0}$ & 20 a 150 & Fuerte \\
\hline 3 & 45.2749 & 13.4717 & 3.4000 & $\underline{0.9654}$ & Más de 150 & Muy fuerte \\
\hline
\end{tabular}

El número de rezagos va de cero a tres y el número de estados de uno a cuatro para la ecuación (1). El logaritmo base 10 del factor de Bayes se estimó en relación con la especificación de más alta probabilidad marginal, marcada en negrita. 


\section{Resultados}

Con el objeto de presentar y contextualizar los resultados de la estimación trimestral del PIB en los periodos siguientes fuera de la muestra, en la primera subsección de esta sección se ofrecen las inferencias asociadas a la especificación markoviana de mejor ajuste a los datos desarrollada en la sección anterior, y en la segunda parte analizamos las funciones de impulso-respuesta bajo la incertidumbre de los regímenes y la aplicación de shocks negativos tanto persistentes como sólo para el primer periodo de tiempo fuera de la muestra. Los códigos de cálculo utilizados para esta sección son propios y se pueden proporcionar a quien lo solicite a los autores.

\subsection{Inferencia asociada al modelo autorregresivo markoviano}

Con base en la propuesta presentada en la sección anterior, la especificación del modelo autorregresivo para la tasa trimestral de crecimiento del PIB de México de Q1-1960 a Q4-2019 quedaría como:

$$
\begin{array}{r}
y_{t}=\zeta_{\left[S_{t}\right]}+\phi_{1\left[S_{t}\right]} y_{t-1}+\phi_{2\left[S_{t}\right]} y_{t-2}+\sigma_{\left[S_{t}\right]} \varepsilon_{t} \\
\text { donde } \varepsilon_{t} \sim \operatorname{NIID}(0,1) \text { y } S_{t}=\{1,2,3,4\}
\end{array}
$$

La estimación de la ecuación (2) y las inferencias asociadas de esta sección se llevan a cabo por simulación Monte-Carlo restringida; es decir, se impone la identificación de cuatro estados con cuatro diferentes volatilidades y medias dependientes del estado. De dicha estimación es posible inferir la media y la volatilidad posterior del proceso en cada régimen, así como las probabilidades suavizadas que indicarían el cambio de régimen. Cuando las probabilidades suavizadas alcanzan un valor cercano a uno el régimen prevalece; en cambio, cuando disminuyen se incrementa la posibilidad de un cambio de régimen.

En la figura 2 se ilustran la temporalidad específica de los cuatro estados definidos por su media en tasa de crecimiento y la volatilidad de la misma. Así, por ejemplo, el régimen (1) de recesión profunda con la volatilidad más alta (3.0779) se presenta en las crisis de 1995 y 2008-2009. De 1980 a 1991 y en periodos posteriores a la recesión de 1995 se tiene evidencia del régimen (2), de crecimiento medio (0.7252) con volatilidad media (1.4389). En el régimen (3), trimestres anteriores a la recesión de 1995 y de 1998 a 2019, excepto la recesión de 2008-2009 y un trimestre de alto crecimiento en 2009, el PIB de México ha permanecido en un régimen de más bajo crecimiento (0.6432) con volatilidad baja (0.6219), lo que caracteriza al periodo más reciente. Finalmente, el régimen (4), de tasa media de crecimiento alto (1.5905) con la volatilidad más baja (0.5749), caracterizó al PIB de México de 1960 a 1980. Sin embargo, este régimen no se vuelve a presentar con una probabilidad importante en el resto de la muestra. Por lo tanto, se consideraría que hubo un cambio estructural en la tasa de crecimiento del PIB a partir de 1980, y en lo que sigue ya no se tomaría en cuenta. 
(1) Recesión y volatilidad alta

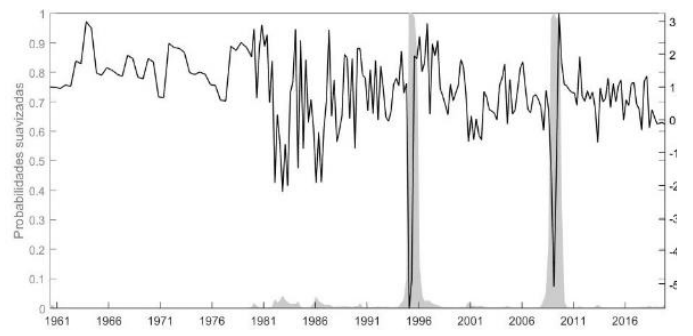

Media:-0.8959, volatilidad: 3.0779

(3) Crecimiento bajo y volatilidad baja

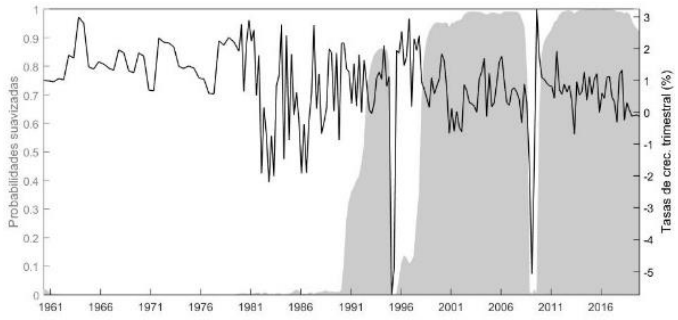

Media: 0.6219 , volatilidad: 0.6432
(2) Crecimiento medio y volatilidad media

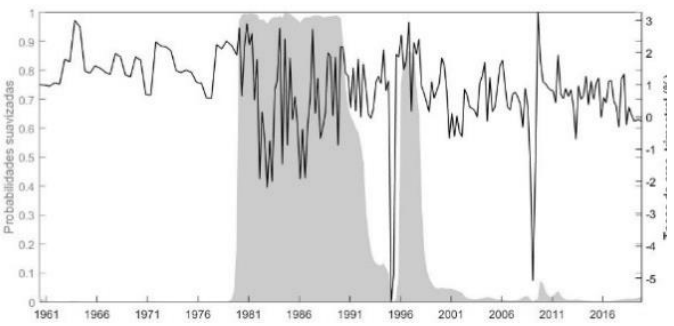

Media: 0.7252, volatilidad: 1.4389

(4) Crecimiento alto y volatilidad baja

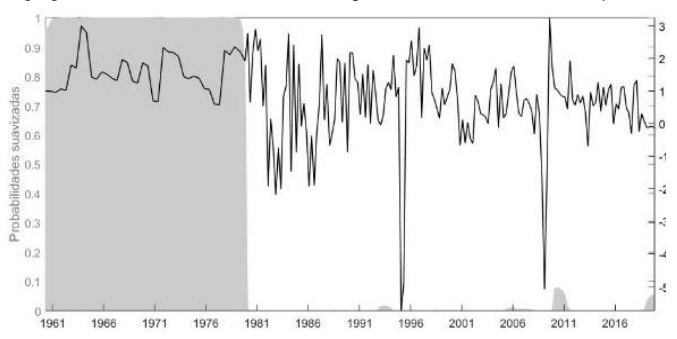

Media: 1.5905, volatilidad: 0.5749

Figura 2. Probabilidades suavizadas y los cuatro estados para el crecimiento del PIB, 1960-2019

El área gris representa las probabilidades suavizadas de cada estado. La media de la tasa de crecimiento y la volatilidad por estado se estimaron con la media posterior de los parámetros de la ecuación (2) y las ecuaciones Yule-Walker.

En resumen, dada la memoria de la serie de tiempo identificada utilizando nuestro procedimiento, sólo se han presentado tres regímenes desde 1980 hasta 2019, y son los únicos que podrían presentarse con alguna probabilidad importante a partir de 2020. En el análisis siguiente para evaluar los impactos de las perturbaciones o shocks en la inferencia fuera de la muestra debido a las funciones de impulso-respuesta de la tasa de crecimiento del PIB, sólo consideramos los regímenes de recesión profunda con volatilidad alta, de crecimiento medio con volatilidad media y de crecimiento bajo (que pudiera mostrar estancamiento económico) con volatilidad baja.

En la Tabla 2 reportamos las probabilidades de permanencia de cada estado estacionario: 9\% de recesión profunda, $26 \%$ de crecimiento medio y $65 \%$ de crecimiento bajo aproximadamente. Así mismo, las probabilidades de transición entre los estados muestran que el estado más persistente es el de bajo crecimiento con $97 \%$ y el menos persistente es el de recesión profunda con $79 \%$ aproximadamente. Las transiciones más significativas son cercanas al 11\% (de recesión a crecimiento medio y de recesión a crecimiento bajo). 
Tabla 2. Probabilidades de transición y estado estacionario de tres estados

\begin{tabular}{|c|c|c|c|c|c|}
\hline Transición & $\mathbf{1}$ & $\mathbf{2}$ & $\mathbf{3}$ & \multicolumn{2}{|c|}{ Estado estacionario } \\
\hline 1 & 0.7855 & 0.1053 & 0.1092 & $\mathrm{Pr}_{1}$ & 0.0886 \\
\hline 2 & 0.0108 & 0.9495 & 0.0397 & $\mathrm{Pr}_{2}$ & 0.2623 \\
\hline 3 & 0.0249 & 0.0060 & 0.9690 & $\mathrm{Pr}_{3}$ & 0.6491 \\
\hline
\end{tabular}

Sólo se presentan la estimación posterior de la matriz de transición de tres estados y sus probabilidades estacionarias (última columna).

\subsection{Análisis de las funciones de impulso-respuesta}

En esta subsección se presentan los resultados de la estimación del valor esperado de la introducción de shocks en la tasa trimestral de crecimiento del PIB tomando en cuenta la incertidumbre de los regímenes en funciones de impulso-respuesta (IRF), con el objeto de estudiar la inferencia fuera de la muestra. La metodología aplicada aquí es similar a la propuesta por Karamé (2010) y Hafner y Herwartz (2006).

Dados los objetivos que intentamos alcanzar en este trabajo, es relevante mencionar que los modelos autorregresivos markovianos dependen de su propia dinámica en el tiempo y que pueden cambiar tanto en su media como en su volatilidad en tres estados diferentes (recuérdese que el estado de alto crecimiento y baja volatilidad representó en 1980 un cambio estructural en la tasa de crecimiento del PIB de México, por lo que es muy baja la probabilidad de que vuelva suceder). Para lograr este objetivo, los resultados de la Tabla 2 son importantes para obtener las ponderaciones que se aplicarán a cada inferencia de las IRF.

Por ejemplo, en una inferencia fuera de la muestra, con una probabilidad de $65 \%$ podría darse la dinámica de bajo crecimiento con baja volatilidad, con una probabilidad del $9 \%$ la de recesión profunda y alta volatilidad, y la de crecimiento medio con volatilidad media con una probabilidad de $26 \%$. Tomando estas probabilidades como ponderadores y los resultados de la estimación de la ecuación (2) por estado como sendas dinámicas, es posible estimar el valor esperado de la tasa de crecimiento del PIB. Siguiendo con el mismo ejemplo, se introduce un shock negativo del 8\%, es decir $\varepsilon_{T+1}=-8$, y se estima el valor esperado del crecimiento del PIB para el primer periodo fuera de la muestra.

Para el segundo periodo de la muestra habría nueve sendas posibles: de recesión a cualquiera de los otros tres estados, de crecimiento medio a cualquiera de los tres, y de crecimiento bajo a los otros tres. Por ejemplo, la probabilidad de estar en recesión en $T+1$ y seguir en recesión en $T+2$ sería $0.0886(0.7855)=0.0696$. De esta manera se podría calcular la probabilidad de cada senda y estimar el valor esperado del crecimiento del PIB en $T+2$.

A partir de este análisis la pregunta importante sería ¿qué tipo de shock se debería aplicar en el próximo periodo de tiempo? Algunas respuestas podrían ser: -8\% igual que el inicial, un shock aleatorio con media de cero para el término de error, shocks subsecuentes negativos o positivos, o simplemente, como lo sugiere la literatura estándar, shocks de cero después del segundo periodo de tiempo fuera de la muestra. Para elegir una respuesta nos enfocamos en el tema central de este artículo: los efectos de la crisis sanitaria del covid-19 en la tasa de crecimiento trimestral del PIB, así como en el concepto de shocks realistas, analizado por Hafner y Herwartz (2006). 
La literatura tradicional sugiere que los futuros shocks (después del primer periodo) deberían tomar el valor de cero porque es el valor esperado del término de error. Sin embargo, la crisis sanitaria del covid-19 ha dejado que variables exógenas no consideradas en el modelo, 6 como la recesión de Estados Unidos, que deja un efecto recesivo en México de tres trimestres en promedio (véase Cabrera y De León Arias, 2020), la tasa de desempleo en México, el cierre de actividades económicas, la inestabilidad financiera y política, rebrotes del covid-19 y muchos otros factores, podrían dejar dos diferentes escenarios en los futuros shocks que afectarán la tasa de crecimiento del PIB trimestral en México:

1. Una serie de shocks negativos de manera decreciente a partir del shock inicial. Como también éste es de magnitud incierta, consideramos que habrá shocks iniciales negativos del 2\% al 8\% en el primer trimestre fuera de la muestra.

2. Aplicación de políticas estabilizadoras por parte del Gobierno de México, o algunos otros eventos similares en cuanto a su papel estabilizador, que podrían anular los shocks negativos futuros y dejarían una variable aleatoria con media de cero después del segundo periodo.

Continuando con el ejemplo anterior, en el primer caso se aplicarían shocks negativos de 8\% a $6 \%, 4 \%, 2 \%$ y $0 \%$. De este periodo en adelante se le considera con la media de cero del término de error, y así quedaría hasta el final de la inferencia de 12 trimestres. En el segundo caso, asumiendo que el Gobierno de México instrumente políticas estabilizadoras que pudieran contrarrestar los shocks negativos derivados de la crisis sanitaria del covid-19, los shocks pasarían a ser una variable aleatoria con media de cero y volatilidad asociada a cada régimen. Este sería el escenario más optimista. Siguiendo con el ejemplo, se aplicaría un shock negativo del 8\%, y del segundo trimestre en adelante los shocks regresarían a una variable aleatoria con media de cero asociada a la volatilidad, lo que dependería del cambio de régimen en la tasa de crecimiento del PIB.

Así, en lo que sigue, se analiza el escenario 1 de la siguiente manera:

1. Se consideran cuatro diferentes shocks negativos de $2 \%$ a $8 \%$ en el primer periodo de tiempo fuera de la muestra, es decir, en $T+1$.

2. Así, en $T+1$ habrá tres posibles sendas del crecimiento del PIB: recesión con alta volatilidad, crecimiento medio con volatilidad media y crecimiento bajo con volatilidad baja. Por lo tanto, la magnitud final de cada shock negativo dependerá de la volatilidad asociada a cada estado. Finalmente, el valor esperado del crecimiento del PIB en $T+1$ se obtiene aplicando las probabilidades de estado estacionario de cada régimen como pesos para cada senda (véase la última columna de la Tabla 2).

3. En $T+2$ los shocks negativos se reducen en $2 \%$ con la volatilidad asociada a cada estado, por lo cual hay $3^{2}=9$ sendas posibles del crecimiento del PIB. El valor esperado del efecto en el crecimiento del PIB en $T+2$ se obtiene a partir de las sendas ponderadas por los pesos o las ponderaciones dadas por las probabilidades de estado estacionario multiplicadas por las

\footnotetext{
${ }^{6}$ Por lo tanto, la exclusión de variables afectaría por medio del término de error al modelo. Si se añaden estos determinantes como variables explicativas sus valores para inferencia fuera de la muestra también deberán ser inferidos, por lo que los errores de inferencia también serán asociados al término de error.
} 
probabilidades de transición asociadas por los cambios de régimen de $T+1$ a $T+2$ (véase la Tabla 2).

4. De $T+3$ a $T+12$, es decir, $s=3, \ldots, 12$, los shocks negativos se siguen reduciendo en $2 \%$, y en caso de llegar a $0 \%$ se considera que el shock de este momento en adelante será aleatorio con media de cero y la volatilidad asociada de cada estado, por lo cual se tendrán $3^{s}$ sendas posibles de crecimiento del PIB. El valor esperado del efecto en el crecimiento del PIB en $T+$ $s$ se obtiene a partir de las sendas aplicando los pesos dados en $T+s-1$ multiplicadas por las probabilidades de transición asociadas a los cambios de régimen a $T+s$.

Para ilustrar lo anterior presentamos la figura 3. En particular, en el gráfico A de la misma se muestra el resultado de aplicar un shock negativo de $2 \%$ en el primer periodo de tiempo, del segundo en adelante los shocks se aplicaron dada su media de cero. En estas condiciones, nuestras estimaciones muestran que la recesión en la tasa de crecimiento duraría dos trimestres; sin embargo, este podría ser un escenario muy poco probable porque se ha reportado una baja de casi el $4 \%$ en el PIB de Estados Unidos, lo cual implicaría una contracción similar en México, pero con mayor persistencia, tomando en cuenta los resultados de Cabrera y De León Arias (2020). El gráfico B ilustra la aplicación de shocks negativos de 4\% y 2\% en los dos primeros trimestres, en este escenario la recesión esperada duraría de tres a cuatro trimestres. En el gráfico C los shocks negativos son de 6\%, $4 \%$ y $2 \%$ en los primeros tres trimestres, por lo que sería de esperar una recesión cercana a los seis trimestres. Finalmente, en el cuarto escenario (D), qué es el más pesimista, los shocks negativos aplicados persisten durante un año y serían siete los trimestres de recesión esperada. ¿Cuál es el escenario más factible? Los datos de diversas fuentes anuncian contracciones en el PIB de entre 4\% y $6 \%$ trimestral; por lo tanto, a éstas se les considera como la magnitud del shock, la recesión esperada duraría de tres a seis trimestres y tendría la forma de U alargada (véanse los gráficos B y C).

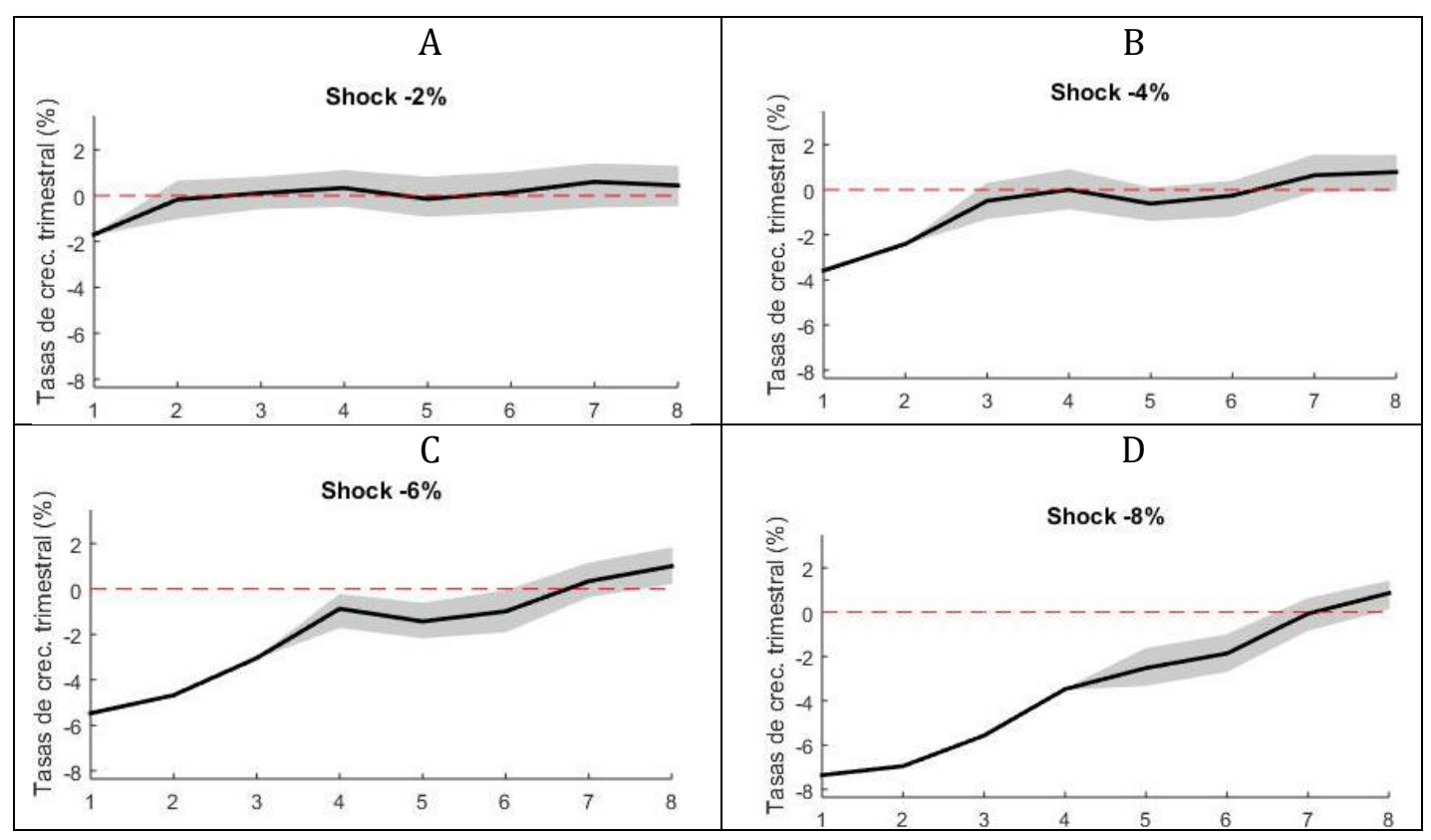

Figura 3. IRF para shocks negativos persistentes

Las bandas de error de las IRF fueron obtenidas por simulación Monte Carlo y shocks aleatorios. 
Desarrollando el escenario 2 de igual manera que con el procedimiento anterior, en las IRF los shocks negativos sólo se aplican en el primer periodo de tiempo, pues se supone que algunas posibles políticas estabilizadoras de México anularían el impacto de shocks futuros y dejarían una variable aleatoria con media de cero en los trimestres posteriores. Este es un escenario relativamente más optimista comparado con el descrito anteriormente.

En este segundo escenario presentamos los shocks negativos en el primer periodo de tiempo, son del $2 \%$ al $8 \%$, para después considerar políticas estabilizadoras que dejarían shocks aleatorios con media de cero a 12 trimestres. Los resultados se ilustran en la figura 4. En el gráfico A de ella se observa que si se estima inicialmente un shock negativo del $2 \%$ la recesión sólo duraría un trimestre. En el gráfico B, con la introducción de un shock de 4\% la recesión duraría poco más de dos trimestres. En el C, con un shock de 6\% la recesión esperada podría durar hasta cuatro trimestres. Por último, en el gráfico D se observa que por un shock de $8 \%$ la recesión esperada podría alcanzar los seis semestres de duración.

En este último análisis, ante shocks negativos de $4 \%$ y 6\%, la recesión esperada sería de dos o tres trimestres en forma de $\mathrm{V}$ y podría llegar a cinco en forma de $\mathrm{W}$ (véanse los gráficos $\mathrm{B}$ y $\mathrm{C}$ de la figura 4).

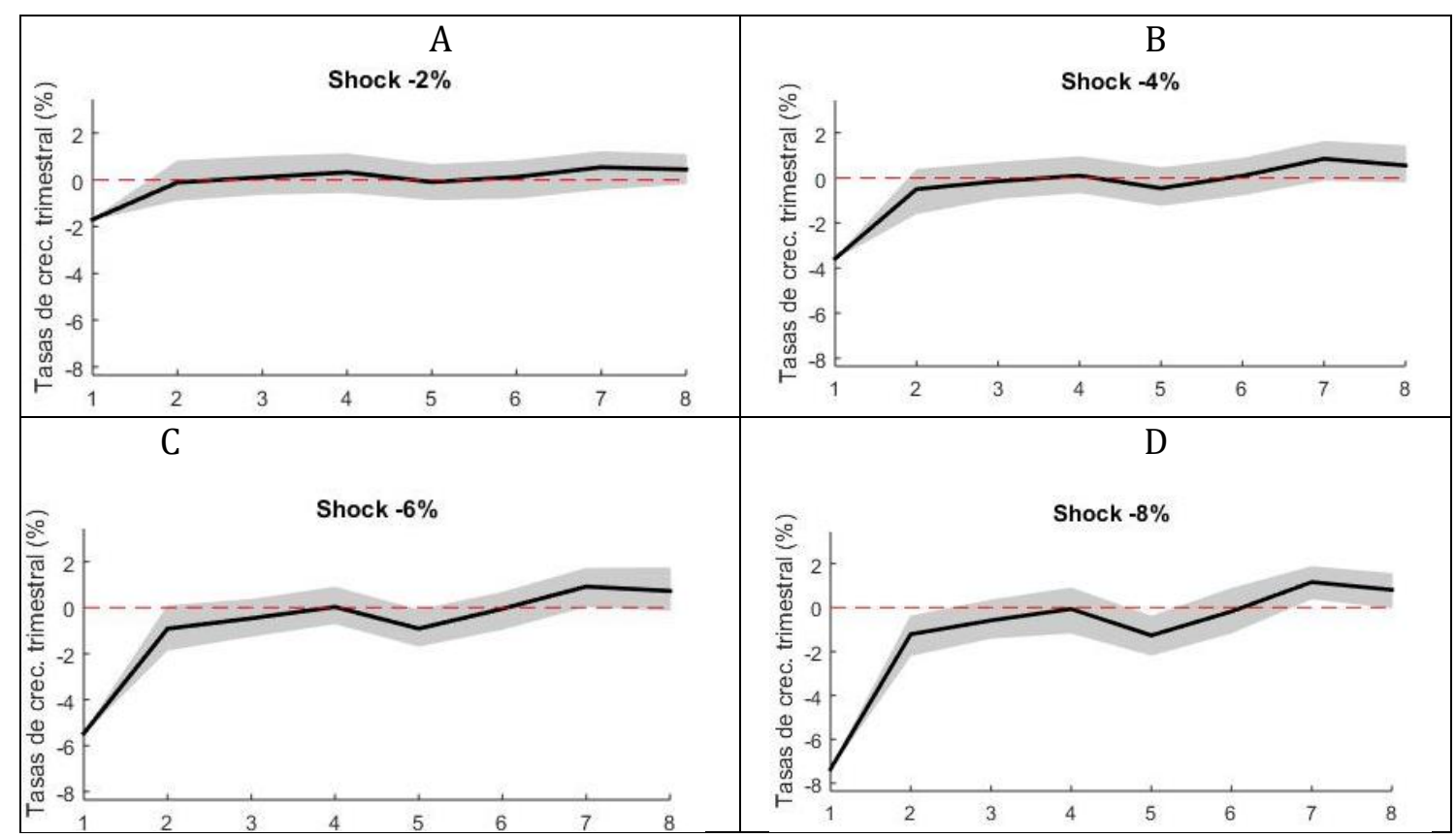

Figura 4. IRF para shocks negativos y políticas estabilizadoras

Las bandas de error de las IRF fueron obtenidas por simulación Monte Carlo y shocks aleatorios.

\section{Conclusiones}

En la primera parte de nuestro análisis identificamos los estados o regímenes de la tasa de crecimiento del PIB de México de Q1-1960 a Q4-2019. Con base en nuestros resultados concluimos que hubo un cambio estructural en la dinámica de la tasa de crecimiento del PIB en 1980, pues de 1960 a 1980 la tasa de crecimiento promedio fue la más alta de la muestra, lo cual estuvo asociado a 
una baja volatilidad. Desde dicho año hasta la fecha, la media de crecimiento del PIB no ha vuelto a alcanzar dicho nivel de expansión y la volatilidad ha presentado cambios en el tiempo. Por otro lado, de 1997 a 2019, excepto en algunos trimestres de 2008 y 2009, la tasa de crecimiento promedio se ha caracterizado primordialmente por ser la más baja de la muestra, lo que se asocia con una baja volatilidad.

Luego de nuestro primer resultado, una vez identificados tres regímenes o estados de la dinámica de crecimiento del PIB trimestral de 1980 a 2019, estudiamos los efectos de dicha tasa de crecimiento trimestral para doce periodos fuera de la muestra, de los que evaluamos los efectos de respuesta esperados ante shocks negativos de diferente magnitud derivados de la crisis sanitaria del covid-19 bajo dos escenarios; en el primero consideramos shocks negativos persistentes debido a la crisis sanitaria; en el segundo, un shock negativo inicial y la aplicación de eventos estabilizadores por el Gobierno de México u otros agentes que anulan las perturbaciones negativas derivadas de dicha crisis, dejando shocks como variable aleatoria con media esperada de cero y volatilidad asociada a cada cambio de régimen esperado en el futuro.

Los efectos esperados de los shocks negativos persistentes en la tasa de crecimiento del PIB anticipados causarían una recesión que duraría de tres a seis trimestres, lo cual dependería de la magnitud negativa del shock inicial del 4\% al 6\% (para una recuperación en forma de U alargada). Por otro lado, en un escenario optimista, asumiendo que las políticas estabilizadoras de México detuvieran la persistencia de los shocks negativos a partir del segundo trimestre, la recesión anticipada podría durar dos trimestres (para una recuperación en forma de V), o de tres a cinco trimestres (para una recuperación en forma de W), dados shocks iniciales del 4\% y 6\% respectivamente.

Dentro de las limitaciones y extensiones de nuestro trabajo enunciamos las siguientes: Es importante mencionar que no consideramos determinantes exógenos para explicar la tasa de crecimiento del PIB de México, como la tasa de crecimiento del PIB de Estados Unidos, el desempleo y la inestabilidad financiera y política, entre otros. Para todas estas variables explicativas, la inferencia fuera de la muestra del PIB de México implicaría también la inferencia de los determinantes mencionados, lo cual incorporaría los errores de medición al término de error del modelo. Por ejemplo, un sistema de ecuaciones autorregresivo con cambio de régimen sería útil para nuestros propósitos. Sin embargo, las posibles combinaciones de todos los estados entre múltiples series de tiempo para las funciones de impulso-respuesta tendría como resultado una tarea de inferencia complicada (véase Cabrera y De León Arias, 2020, quienes estudian los co-movimientos entre las tasas de crecimiento del PIB de Estados Unidos y México en un VAR markoviano).

Por otro lado, la dinámica de recuperación económica se puede inferir con indicadores de alta frecuencia (datos mensuales como índices de actividad económica, datos diarios del covid-19 y encuestas del Banco de México, entre otras fuentes). Sin embargo, dejamos este análisis de combinación de datos en diferentes frecuencias y pronóstico para investigación futura.

Finalmente, la aplicación de shocks negativos de gran magnitud, como su persistencia, muestra una limitación importante en el término de error sobre la normalidad y la independencia estocástica. Futuras investigaciones deberían considerar el relajamiento de este supuesto en el término de error para su aplicabilidad de acuerdo con la evidencia empírica. 


\section{References}

[1] Baker, S. R., Bloom, N., Davis, S. J. y Terry, S. J. (2020). Covid-induced economic uncertainty. NBER Working Paper Series 26983. https://doi.org/10.3386/w26983.

[2] Boot, T. y Pick, A. (2017). Optimal forecasts from Markov switching models. Journal of Business \& Economic Statistics, 36(4), 628-642. https://doi.org/10.1080/07350015.2016.1219264.

[3] Burns, A. y Mitchell, W. (1946). Measuring business cycles, NBER Books.

[4] Cabrera, G. y De León Arias, A. (2020). Co-movimientos, sincronización y efectos de transmisión entre el PIB de Estados Unidos y México. Documento de trabajo. https://sites.google.com/site/personalgcgnew/home/research/new-working-papers.

[5] Cabrera, G. y De León Arias, A. (2019). Modelación markoviana para identificar la dinámica y pronóstico del índice de producción industrial en México de 1980 a 2018, EconoQuantum, 16(2), 2341. https://doi.org/10.18381/eq.v16i2.7120.

[6] Cabrera, G., Coronado, S., Rojas, O. y Romero-Meza, R. (2018). A Bayesian approach to model changes in volatility in the Mexican stock exchange index. Applied Economics, 50(15), 1716-1724. https://doi.org/10.1080/00036846.2017.1374536.

[7] De León Arias, A. (2004). El crecimiento de México y su interdependencia con Estados Unidos. Comercio Exterior, 54(7), 612-619. https://orcid.org/0000-0002-2209-500X.

[8] Diebold, F. y Rudebusch, G. (1999). Business cycles: durations, dynamics, and forecasting. Princeton University Press.

[9] Fernandes, N. (2020). Economic effects of coronavirus outbreak (covid-19) on the world economy. Working paper. http://dx.doi.org/10.2139/ssrn.3557504.

[10] Frühwirth-Schnatter, S. (2006). Finite mixture and Markov switching models. Springer. https://doi.org/10.1007/978-0-387-35768-3_6.

[11] Frühwirth-Schnatter, S. (2004). Estimating marginal likelihoods for mixture and Markov switching models using bridge sampling techniques. The Econometrics Journal, 7(1), 143-167. https://doi.org/10.1111/j.1368-423X.2004.00125.x.

[12] Günay, S. (2020a). Covid-19 pandemic versus global financial crisis: evidence from currency market. Working paper. https://doi.org/10.2139/ssrn.3584249.

[13] Günay, S. (2020b). A new form of financial contagion: covid-19 and stock market responses, Working paper. https://doi.org/10.13140/RG.2.2.24444.08325.

[14] Hafner, C. M. y Herwartz, H. (2006). Volatility impulse responses for multivariate GARCH models: an exchange rate illustration. Journal of International Money and Finance, 25(5), 719-740. https://doi.org/10.1016/j.jimonfin.2006.04.006.

[15] Hamilton, J. D. (1989). A new approach to the economic analysis of nonstationary time series and the business cycle. Econometrica, 57(2), 357-384. https://doi.org/10.2307/1912559.

[16] Hamilton, J. y Raj, B. (2002). New directions in business cycle research and financial analysis. Empirical Economics, 27 (2), 149-162. https://doi.org/10.1007/s001810100115.

[17] Karamé, F. (2010). Impulse-response functions in Markov-switching structural vector autoregressions: a step further. Economics Letters, 106(3), 162-165. https://doi.org/10.1016/j.econlet.2009.11.009.

[18] Kass, R. E. y Raftery, A. F. (1995). Bayes factors. Journal of the American Statistical Association, 90(430), 773-795. https://doi.org/10.1080/01621459.1995.10476572.

[19] Mejía-Reyes, P., Martínez Gómez, J. A. y Rendón Balboa, W. L. (2005). Ciclos económicos clásicos en la producción industrial de México. Investigación Económica, LXIV(254), 91-124. https://www.redalyc.org/articulo.oa?id=601/60125403. 
[20] Mejía-Reyes, P. y Díaz-Carreño, M. Á. (2010). Búsqueda de asimetrías a lo largo del ciclo económico de México. Investigación $\quad$ Económica, LXIX(271), 15-42. http://www.scielo.org.mx/scielo.php?script=sci_arttext\&pid=S0185-16672010000100002.

[21] Ozili, P. K. y Thankom, A. (2020). Spillover of COVID-19: impact on the global economy. Working paper. http://dx.doi.org/10.2139/ssrn.3562570.

[22] Sulkowski L, (2020). Covid-19 pandemic: recession, virtual revolution leading to de-globalization? Journal of Intercultural Management, 12(1), 1-11. https://doi.org/10.2478/joim-2020-0029.

[23] Zeren, F. y Hizarci, A. (2020). The impact of covid-19 coronavirus on stock markets: evidence from selected countries. Bulletin of Accounting and Finance Reviews, 3(1), 78-84, https://doi.org/10.32951/mufider.706159. 